\title{
Analysis of stromal cells in osteofibrous dysplasia and adamantinoma of long bones
}

\author{
Richard M Taylor ${ }^{1}$, Takeshi G Kashima ${ }^{1}$, David J Ferguson ${ }^{1}$, Károly Szuhai ${ }^{2}$, \\ Pancras C Hogendoorn ${ }^{2}$ and Nicholas A Athanasou ${ }^{1}$ \\ ${ }^{1}$ Nuffield Department of Orthopaedics, Rheumatology and Musculoskeletal Sciences, University of Oxford, \\ Nuffield Orthopaedic Centre, Oxford, UK and ${ }^{2}$ Department of Pathology, Leiden University Medical Centre, \\ Albinusdreef 2, Leiden, The Netherlands
}

\begin{abstract}
Adamantinoma of long bones and osteofibrous dysplasia are rare, osteolytic primary bone tumours of uncertain origin containing areas of fibrous and fibro-osseous proliferation. We investigated the nature of the stromal cells in adamantinoma of long bones and osteofibrous dysplasia, and determined cellular and molecular mechanisms of osteolysis in these tumours. Cell culture, molecular (RT-PCR, western blot) and immunohistochemical studies on cases of adamantinoma of long bones and of osteofibrous dysplasia were undertaken to determine the expression of epithelial, osteoblast and osteoclast markers. Ultrastructural and immunophenotypic studies on cultured adamantinoma and osteofibrous dysplasia stromal cells showed that these cells were mainly fibroblast-like with few cells expressing epithelial markers. Osteofibrous dysplasia but not adamantinoma cells expressed alkaline phosphatase. Both osteofibrous dysplasia and adamantinoma cells expressed the ostoclastogenic factors M-CSF and RANKL. Adamantinoma and osteofibrous dysplasia cells also expressed messenger RNA for osteocalcin, osteonectin, osteopontin, osterix and collagen type 1. Adamantinoma and osteofibrous dysplasia cells cultured alone on dentine slices were not capable of lacunar resorption, but in co-cultures with monocytes induced formation of osteoclast-like cells was observered. Cultured osteofibrous dysplasia and adamantinoma stromal cells show similar ultrastructural and immunophenotypic characteristics, and differentially express osteoblast markers. Promotion of osteoclastogenesis by stromal cells may contribute to osteolysis in adamantinoma of long bones and osteofibrous dysplasia. Modern Pathology (2012) 25, 56-64; doi:10.1038/modpathol.2011.141; published online 7 October 2011
\end{abstract}

Keywords: adamantinoma of long bones; bone tumour; osteoclast; osteofibrous dysplasia

Adamantinoma of long bones is a rare primary malignant skeletal neoplasm that is composed of epithelial and mesenchymal elements. ${ }^{1,2}$ Osteofibrous dysplasia is a fibro-osseous proliferative lesion which, like adamantinoma of long bones, most commonly affects the tibia and fibula. ${ }^{3}$ Osteofibrous dysplasia is most often a self-limited process and typically occurs during the first two decades of life. Adamantinoma of long bones most commonly affects patients $>20$ years but may develop at an earlier age. Adamantinoma of long bones and osteofibrous dysplasia are both lytic tumours, producing multiloculated radiolucent expansion of

Correspondence: Professor NA Athanasou, MD, PhD, Nuffield Department of Orthopaedics, Rheumatology and Musculoskeletal Sciences, University of Oxford, Nuffield Orthopaedic Centre, Oxford OX3 7LD, UK.

E-mail: nick.athanasou@ndorms.ox.ac.uk

Received 22 February 2011; revised 5 July 2011; accepted 5 July 2011; published online 7 October 2011 the anterolateral cortex of the tibia with associated anterior bowing, cortical thickening and sclerosis of the mid shaft.

Adamantinoma of long bones is characterised histologically by the presence of a prominent epithelial component as well as areas of osteofibrous dysplasia-like fibro-osseous tissue. ${ }^{1,2,4}$ In differentiated adamantinoma of the tibia, which usually occurs at an earlier age and has a low metastatic potential, osteofibrous dysplasia-like areas are very prominent. Most cases of osteofibrous dysplasia contain scattered cells that express an epithelial phenotype. $^{4-7}$ Osteofibrous dysplasia may also contain areas of haemorrhage and collections of multinucleated giant cells. The clinical, radiological and histological similarities between osteofibrous dysplasia and adamantinoma of long bones suggest that these two lesions may be related. ${ }^{1,2}$ Cytogenetic studies have revealed a number of chromosomal abnormalities in osteofibrous dysplasia and adamantinoma of long bones, ${ }^{1,6}$ and it has been 
proposed that osteofibrous dysplasia may represent a precursor lesion of adamantinoma of long bones. ${ }^{8,9}$ It has also been postulated that osteofibrous dysplasia is the end result of a reparative process that represents regression of adamantinoma of long bones. ${ }^{10}$

As fibrous and fibro-osseous tissue is a prominent feature in both osteofibrous dysplasia and adamantinoma of long bones, we sought to determine the nature of the fibroblastic stromal cells in these two lesions. We have examined the cell culture characteristics of stromal cells isolated from osteofibrous dysplasia and adamantinoma of long bones, and analysed expression of markers of epithelial, osteoblast and osteoclast differentiation to determine whether the stromal cells express similar phenotypic characteristics. As both osteofibrous dysplasia and adamantinoma of long bones are osteolytic tumours, we also assessed whether stromal cells in these lesions are capable of directly effecting osteolysis, and whether they express factors that promote osteoclast formation and resorption.

\section{Materials and methods}

\section{Adamantinoma of Long Bones and Osteofibrous Dysplasia Cases Examined}

Two cases of adamantinoma of long bones involving the tibia, one a classic adamantinoma arising in a 55-year-old female, the other a differentiated (osteofibrous dysplasia-like) adamantinoma in a 23-year-old female, and two cases of tibial osteofibrous dysplasia in a 15- and 10-year-old females were analysed in this study. Portions of the tumour were obtained fresh, snap frozen and stored in liquid nitrogen for molecular genetic studies. Representative samples of osteofibrous dysplasia and adamantinoma of long bones were also fixed in formalin for routine histological processing and immunohistochemistry or in glutaraldehyde for ultrastructural studies. The remaining (unfixed) tissue was used for cell culture studies.

\section{Immunohistochemistry}

Immunohistochemistry was carried out on serial sections ( $5 \mu \mathrm{m})$ of adamantinoma of long bones and osteofibrous dysplasia. The presence of cytokeratin intermediate filaments and epithelial membrane antigen (EMA) was sought using monoclonal antibodies AE1/3 and E29 (both derived from Dakopatts). Expression of CD45 (leucocyte common antigen) and CD51, an osteoclast-associated marker, ${ }^{11,12}$ was sought using PD7/26 (Dakopatts, UK) and NCL-CD51 (Novocastra, UK). RANKL expression was also determined using a polyclonal antihuman RANKL antibody (sc-7627, Santa Cruz Biotechnology, USA). Tissue sections were dewaxed and rehydrated by successive immersion in xylene, graded ethanol and water. Antigen retrieval was performed by microwave treatment $(700 \mathrm{~W}$, $2 \times 4 \mathrm{~min}$ ) in Target Retrieval Solution (Dako). Endogenous peroxidise was blocked by $0.2 \%(\mathrm{v} / \mathrm{v})$ hydrogen peroxide in $80 \%$ ethanol and protein block serum before $30 \mathrm{~min}$ incubation with the monoclonal antibodies. Antigen expression was detected by incubation with labelled polymer (ChemMate Envision, Dakopatts, UK) and diaminobenzidine. The sections were counterstained with haematoxylin, dehydrated, cleared and mounted.

\section{Cell Cultures of Adamantinoma of Long Bones and Osteofibrous Dysplasia Stromal Cells}

Fragments of adamantinoma and osteofibrous dysplasia tissue, $\sim 2 \mathrm{~mm}$ in diameter, were placed in Falcon tissue culture flasks and covered with a small amount of minimal essential medium containing $10 \%$ fetal calf serum. Cell cultures of adamantinoma and osteofibrous dysplasia stromal cells were incubated at $37^{\circ} \mathrm{C}$ in a humidified atmosphere containing $5 \% \quad \mathrm{CO}_{2}$, and the culture medium changed after $24 \mathrm{~h}$ and then at 5-7 day intervals. ${ }^{13-15}$ The cultures were continuously observed and the morphology of cells growing out from the fragments was noted. Cultures were then passaged after 6 weeks and then regrown a further 2 weeks.

Passage 3 cells were analysed for expression of alkaline phosphatase by histochemistry and for the ability of the stromal cells to form mineralised nodules in the presence of $\beta$ glycerophosphate and ascorbate using Alizarin Red $S$, as previously described. ${ }^{13,14}$ Cultured cells were also fixed in formalin and assessed immunohistochemically for the expression of cytokeratin, EMA, RANKL, CD45 and CD51, as detailed above. Cultured adamantinoma and osteofibrous dysplasia cells were also fixed in glutaraldehyde and processed for transmission electron microscopy.

To assess whether adamantinoma of long bones and osteofibrous dysplasia stromal cells were capable of resorption, passaged cells were added to dentine slices and glass coverslips in 96-well tissue culture plates containing minimal essential medium/fetal calf serum and then transferred into the 24-well tissue culture plates containing $1 \mathrm{ml}$ minimal essential medium/fetal calf serum, M-CSF $(25 \mathrm{ng} / \mathrm{ml})$ and soluble RANKL (30 ng/ml). Cultures were also set up in the absence of M-CSF and/or RANKL. In addition, cultured adamantinoma and osteofibrous dysplasia stromal cells were incubated with human monocytes obtained from buffy coats, as previously described. ${ }^{15}$ After 14 and 21 days incubation, cultures on glass coverslips were fixed and stained cytochemically for the osteoclast-associated enzyme tartrate-resistant acid phosphatase (TRAP), and immunocytochemically for the presence of CD51, the vitronectin receptor. Functional evidence of osteoclast differentiation was sought in cell cultures on dentine slices to determine evidence of lacunar resorption previously described. ${ }^{15}$ 
RT-PCR Studies on Osteofibrous Dysplasia and Adamantinoma of Long Bones Stromal Cell

Messenger RNA (mRNA) expression of the osteoblast-associated factors osteocalcin, osteonectin, osteopontin, osterix and collagen type 1 was determined in cultured osteofibrous dysplasia and adamantinoma stromal cell by RT-PCR. Briefly, RNA was extracted from cultured cells in TRI Reagent (Sigma) and treated with DNase 1 kit (Invitrogen). Normal human osteoblasts (Lot 3F1152, Cambrex BioScience, Walkersville, MD, USA) were cultured according to the manufacture's protocol and employed as positive control. RNA was tested for quality and concentration using a NanoDrop ND1000 spectrophotometer. Single-strand complementary DNA (cDNA) was synthesised using the SuperScript VILO cDNA Synthesis Kit (Invitrogen). RT-PCR was performed with the Rotor-Gene 3000 (Corbett Research) and the Express SYBR GreenER Kit (qPCR Supermix Universal; Invitrogen). The PCR cycling conditions were as follows: $50^{\circ} \mathrm{C} 2 \mathrm{~min}, 95^{\circ} \mathrm{C}$ $15 \mathrm{~s}$ and $60^{\circ} \mathrm{C} 1 \mathrm{~min}$ for 40 cycles. QuantiTect realtime primers were designed by Qiagen (West Sussex, UK). $\beta$-Actin mRNA expression was served as an internal control. The experiments were repeated at least two times. All the samples were amplified in triplicate. One-way analysis of variance test followed by Dunnett's post hoc test was performed in order to compare the data between control human primary osteoblasts and tested cells using the Graphpad Prism (Graphpad Software, La Jolla, CA, USA).

\section{Western Blotting Studies on Osteofibrous Dysplasia and Adamantinoma of Long Bones Stromal Cells}

Protein expression of M-CSF, RANKL and OPG was determined in cultured osteofibrous dysplasia and adamantinoma stromal cells by western blotting, and osteofibrous dysplasia and adamantinoma stromal cells cultures were washed in cold buffer (PBS, $1 \mathrm{mM}$ of EDTA, $1 \mathrm{mM}$ of PMSF) and lysed (RIPA buffer, 5\% Proteinase Inhibitor Cocktail, (Sigma)). The supernatant was collected and the protein concentration was determined using the Pierce BCA Protein Assay Kit (Thermo Scientific, Hampshire, UK). Samples (100 $\mu$ g per lane) were run on a $10 \%$ acrylamide gel and blotted onto an immobilon membrane (Millipore, UK). Membranes were blocked (milk $+0.125 \%$ Tween (Sigma Aldridge) or $5 \%$ filtered BSA (Invitrogen). Membranes were incubated overnight at $4{ }^{\circ} \mathrm{C}$ with primary antibodies specific for either MCSF (1:10 000; ab52864-Abcam) diluted in 1\% filtered BSA, RANKL (1:1000; AB626RandD), OPG (1:250; ab11994-Abcam,) or $\beta$-tubulin (1:1250; T4026-Sigma) diluted in milk. Subsequently, the membranes were washed in PBS/Tween $(0.125 \%)$ and incubated with appropriate secondary antibodies diluted in either 1\% filtered BSA (antirabbit secondary (Thermo Scientific)) or milk (anti- mouse (Thermo Scientific) or anti-goat (RandD)) for $1 \mathrm{~h}$ at room temperature. Membranes were washed in PBS/Tween $(0.125 \%)$ and immunoreactive bands were visualised using an ECL Plus Western Blotting Detection System (Amersham, UK).

\section{Results}

Histological and Ultrastructural Findings in Osteofibrous Dysplasia and Adamantinoma of Long Bones

The two cases of osteofibrous dysplasia and the two cases of adamantinoma of long bones examined in this study showed the typical morphological features of these lesions (Figure 1). Both adamantinoma cases contained a prominent epithelial component in which tumour cells showed EMA and cytokeratin expression; scattered EMA and cytokeratin-expressing cells were also seen in the stromal cell component of osteofibrous dysplasia and adamantinoma of long bones (Figure 2). Epithelial differentiation, including elaboration of desmosomes in the cases of classic adamantinoma of long bones, was confirmed by ultrastructural studies (Figure 3a); this case exhibited a recurrent 46XX, $\mathrm{t}(1 ; 6)(\mathrm{p} ; 1)$ and $\mathrm{t}(2 ; 2)(\mathrm{p} ; 9)$ translocation by COBRA FISH analysis. Thickened woven bone trabeculae, showing increased remodelling with osteoblastic and osteoclastic activity, were present in both osteofibrous dysplasia and adamantinoma of long bones.

\section{Immunophenotypic and Ultrastructural Characteristics of Adamantinoma of Long Bones and Osteofibrous Dysplasia Stromal Cells}

Cultures of both adamantinoma and osteofibrous dysplasia stromal cells showed similar morphological features, being composed of a proliferation of spindle-shaped cells with elongated round or ovoid nuclei. In both adamantinoma and osteofibrous dysplasia cell cultures, a few $(<1 \%)$ of the passaged cells showed evidence of EMA or cytokeratin expression (Figure 4). There was no expression of CD45 (leukocyte common antigen) or CD51 (vitronectin receptor), antigens that are expressed by osteoclasts. ${ }^{11,14}$ Ultrastructural examination of cultured osteofibrous dysplasia and adamantinoma stromal cells showed fibroblast-like cells with prominent rough endoplasmic reticulum and no evidence of epithelial differentiation (Figure $3 \mathrm{~b}$ ).

\section{Adamantinoma of Long Bones and Osteofibrous Dysplasia Stromal Cells Express Osteoblast Markers}

Osteofibrous dysplasia but not adamantinoma stromal cells expressed alkaline phosphatase, an osteoblast marker; both osteofibrous dysplasia and adamantinoma cells when cultured in the presence of $\beta$ glycerophosphate and ascorbate, did not form 

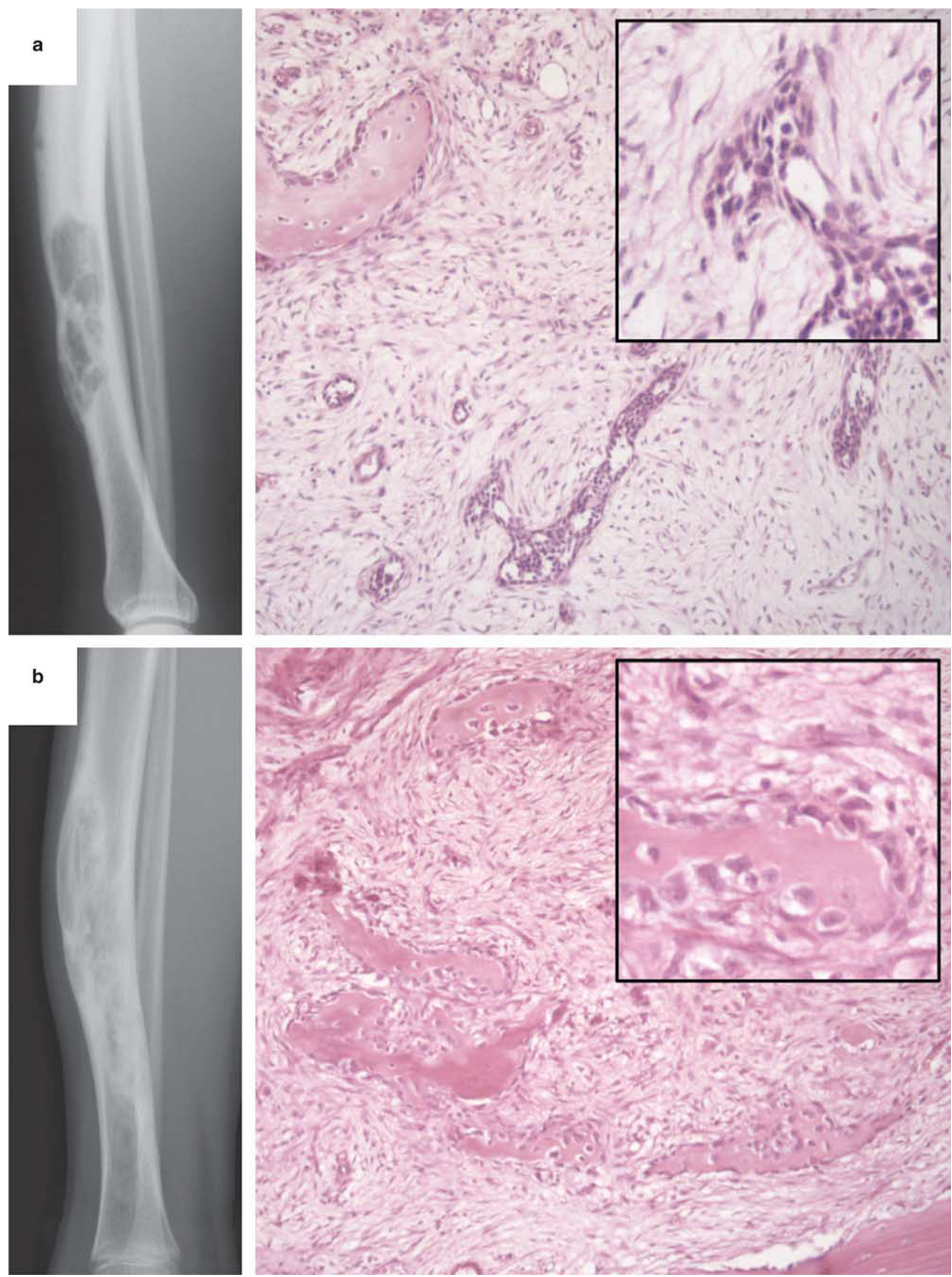

Figure 1 Radiographs and microphotographs of the cases we prepared from the stromal cells. (a) Classic adamantinoma of long bones and (b) osteofibrous dysplasia. 


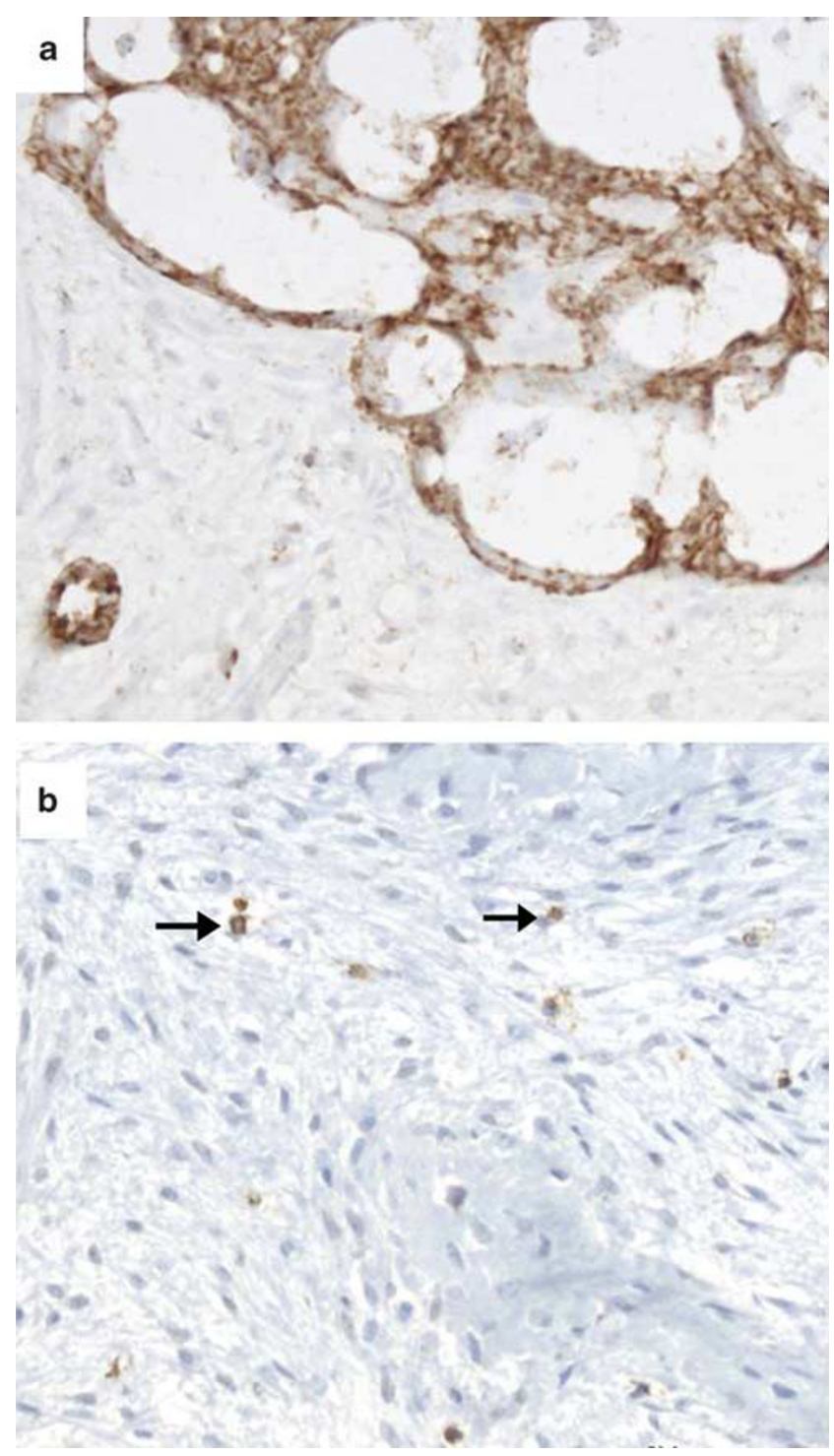

Figure 2 Immunohistochemistry for cytokeratin in (a) classic adamantinoma of long bones and (b) osteofibrous dysplasia (shown by arrows).

mineralised nodules as assessed by Alizarin Red staining.

RT-PCR studies showed expression of RNA transcripts in cultured osteofibrous dysplasia and adamantinoma stromal cells for the osteoblast products osteocalcin, osteonectin, osteopontin, osterix and collagen type 1 (Figure 5).

\section{Adamantinoma of Long Bones and Osteofibrous Dysplasia Stromal Cells and Osteoclastogenesis}

Western blotting showed that cultured adamantinoma and osteofibrous dysplasia stromal cells strongly expressed RANKL, M-CSF and OPG. Two RANKL bands were noted on the membrane, at 45 and $20 \mathrm{kDa}$ indicating the expression of both membranebound and soluble isoforms. Both osteofibrous dysplasia and adamantinoma stromal cells also
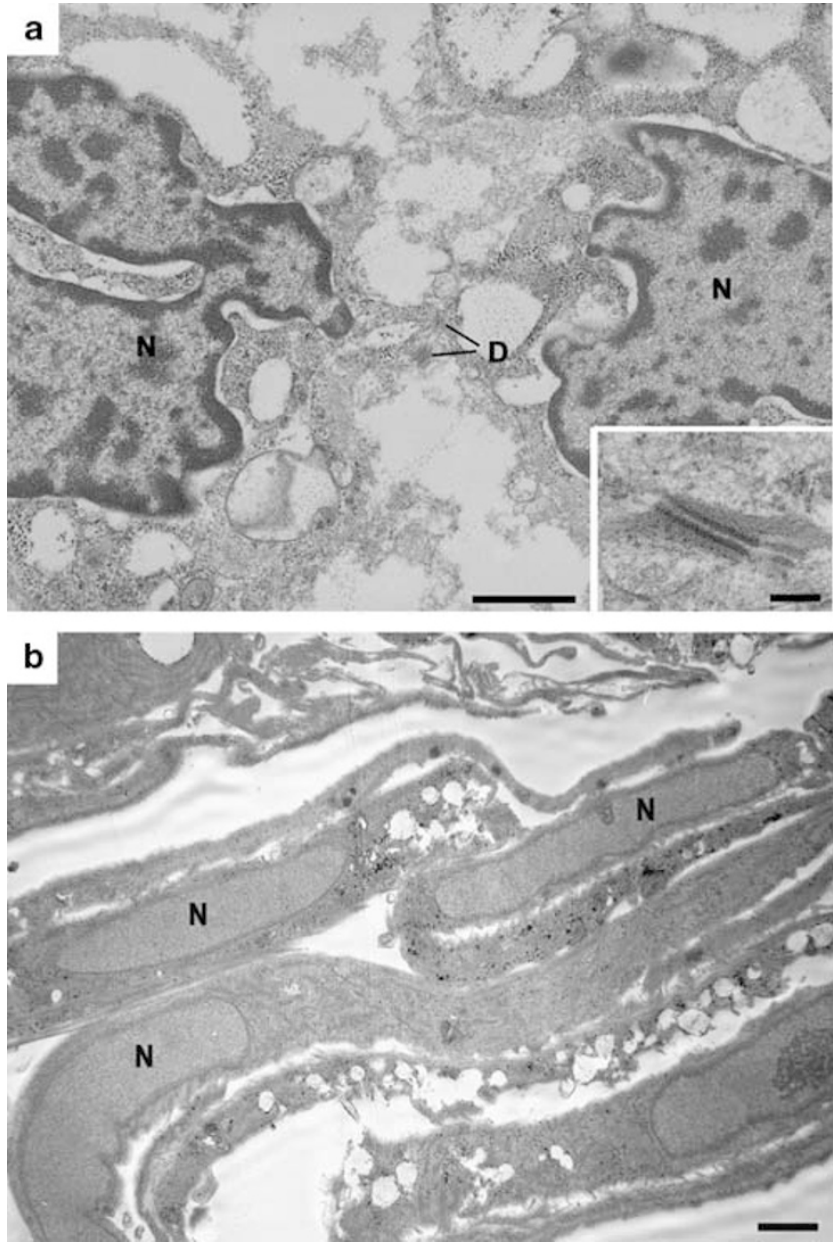

Figure 3 Ultrastructural findings in adamantinoma of long bones. (a) Electron micrograph showing two adamantinoma tumour cells with connecting desmosomes (D). N, nucleus. Scale Bar is $0.5 \mu \mathrm{m}$. Insert, enlargement of a desmosome. Scale Bar is $100 \mathrm{~nm}$. (b) Electron micrograph of cultured adamantinoma stromal cells showing overlapping spindle-shaped cells that lack desmosomes. $\mathrm{N}$, nucleus. Scale Bar is $1 \mu \mathrm{m}$.

expressed the band for OPG (48 $\mathrm{kDa})$ and M-CSF $(60 \mathrm{kDa})$. There was no observed difference in the expression of RANKL, M-CSF or OPG between the cultured osteofibrous dysplasia and adamantinoma stromal cells (Figure 6).

When adamantinoma and osteofibrous dysplasia cells were cultured alone for 21 days on glass coverslips and dentine slices, both in the presence and absence of the osteoclastogenic factors, M-CSF and RANKL, no evidence of lacunar resorption or expression of the osteoclast markers TRAP or CD51 was noted. However, when adamantinoma or osteofibrous dysplasia cells were cultured with human monocytes for up to 21 days, there was evidence of osteoclast differentiation with formation of TRAPpositive multinucleated cells and lacunar resorption (Figure 7a). No evidence of TRAP expression or lacunar resorption was seen in 21-day cultures of monocytes or stromal cells alone (Figures $7 \mathrm{~b}$ and c, respectively). 

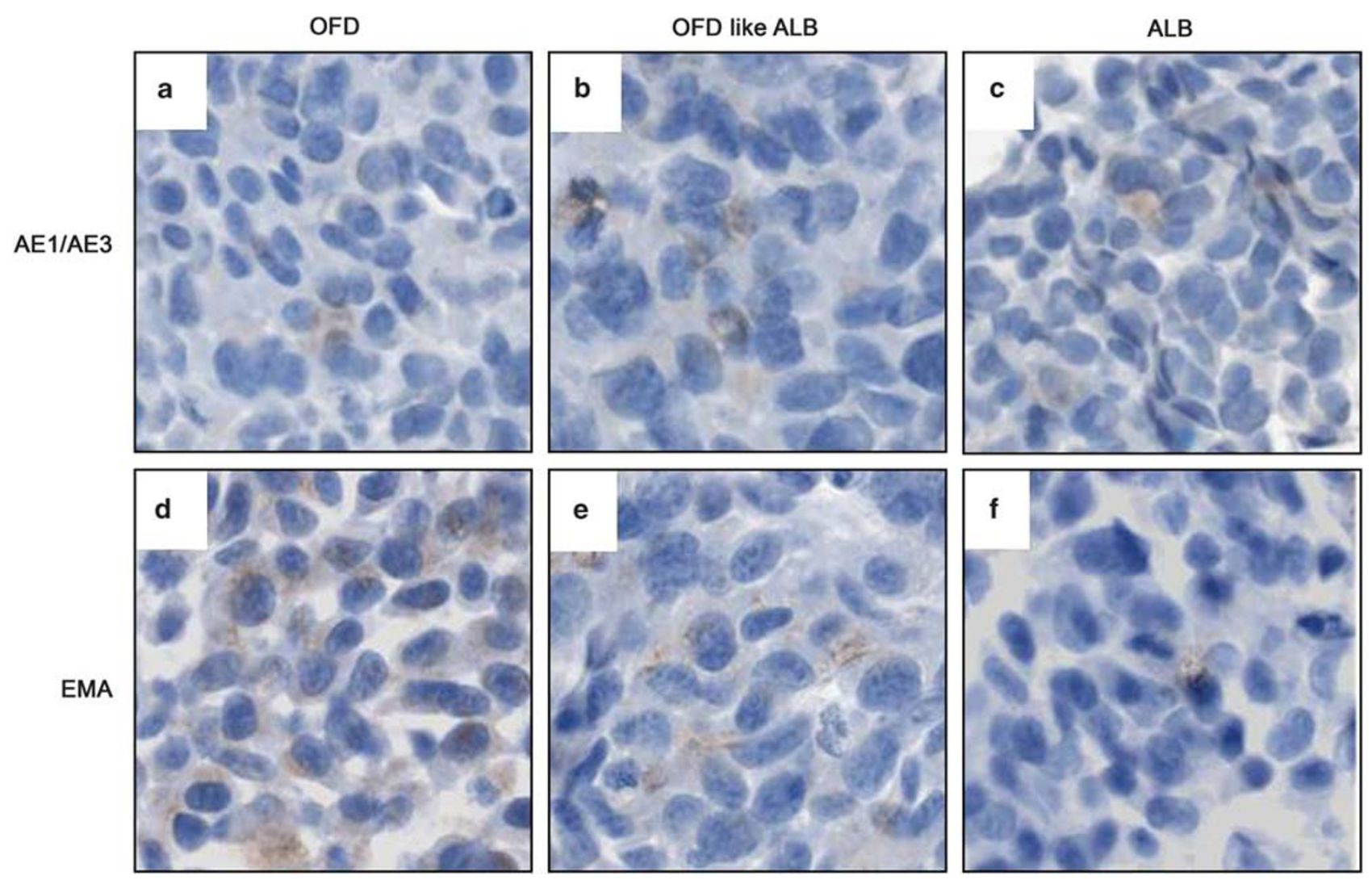

Figure 4 Indirect immunoperoxidase staining of cultured stromal cells derived from osteofibrous dysplasia (OFD), osteofibrous dysplasia-like adamantinoma of long bones (OFD-like adamantinoma of long bones (ALB)) and classic ALB showing (a-c) cytokeratin (AE1/AE3) and (d-f) epithelial membrane antigen (EMA)-expressing cells.

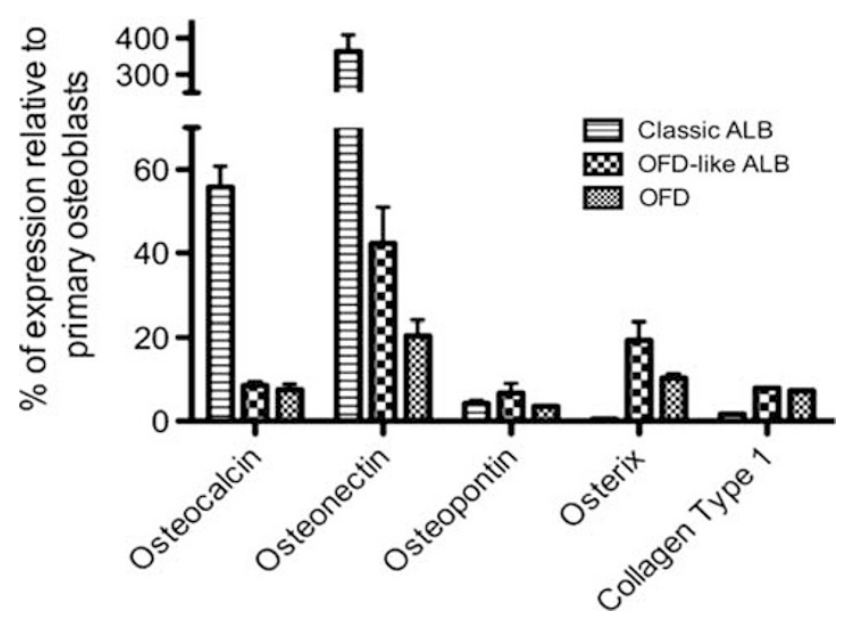

Figure 5 Expression of osteocalcin, osteonectin, osteopontin, osterix and collagen type 1 RNA transcripts by cultured osteofibrous dysplasia/adamantinoma (OFD/ALB) stromal cells. Data normalised to osteoblast control. Mean \pm s.d.

\section{Discussion}

Both adamantinoma of long bones and osteofibrous dysplasia are osteolytic tumours that contain a prominent intraosseous fibrous component. The cell cultures carried out on the cases of adamantinoma of long bones and osteofibrous dysplasia in this study

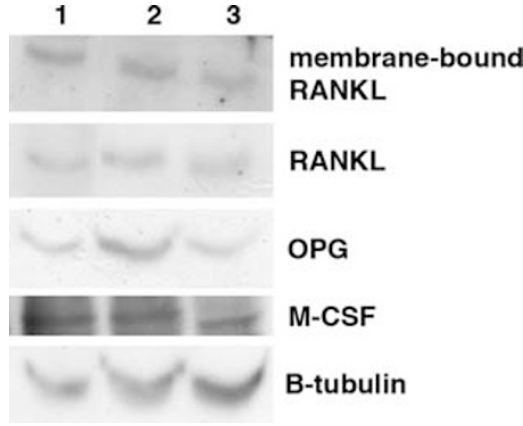

Figure 6 Expression of RANKL, M-CSF and OPG protein by cultured osteofibrous dysplasia/adamantinoma of long bones stromal cells detected by western blotting. Lane 1, adamantinoma of long bones; lane 2, osteofibrous dysplasia; and lane 3, osteofibrous dysplasia-like adamantinoma of long bones.

show that the stromal cells in both these lesions are predominantly mesenchymal in type with very few stromal cells expressing the epithelial markers EMA and cytokeratin. Cultured adamantinoma and osteofibrous dysplasia stromal cells did not exhibit the ultrastructural features of epithelial cells. Osteofibrous dysplasia and adamantinoma stromal cells exhibited some characteristic of the osteoblast phenotype. Osteofibrous dysplasia cells expressed alkaline phosphatase, and osteofibrous dysplasia 

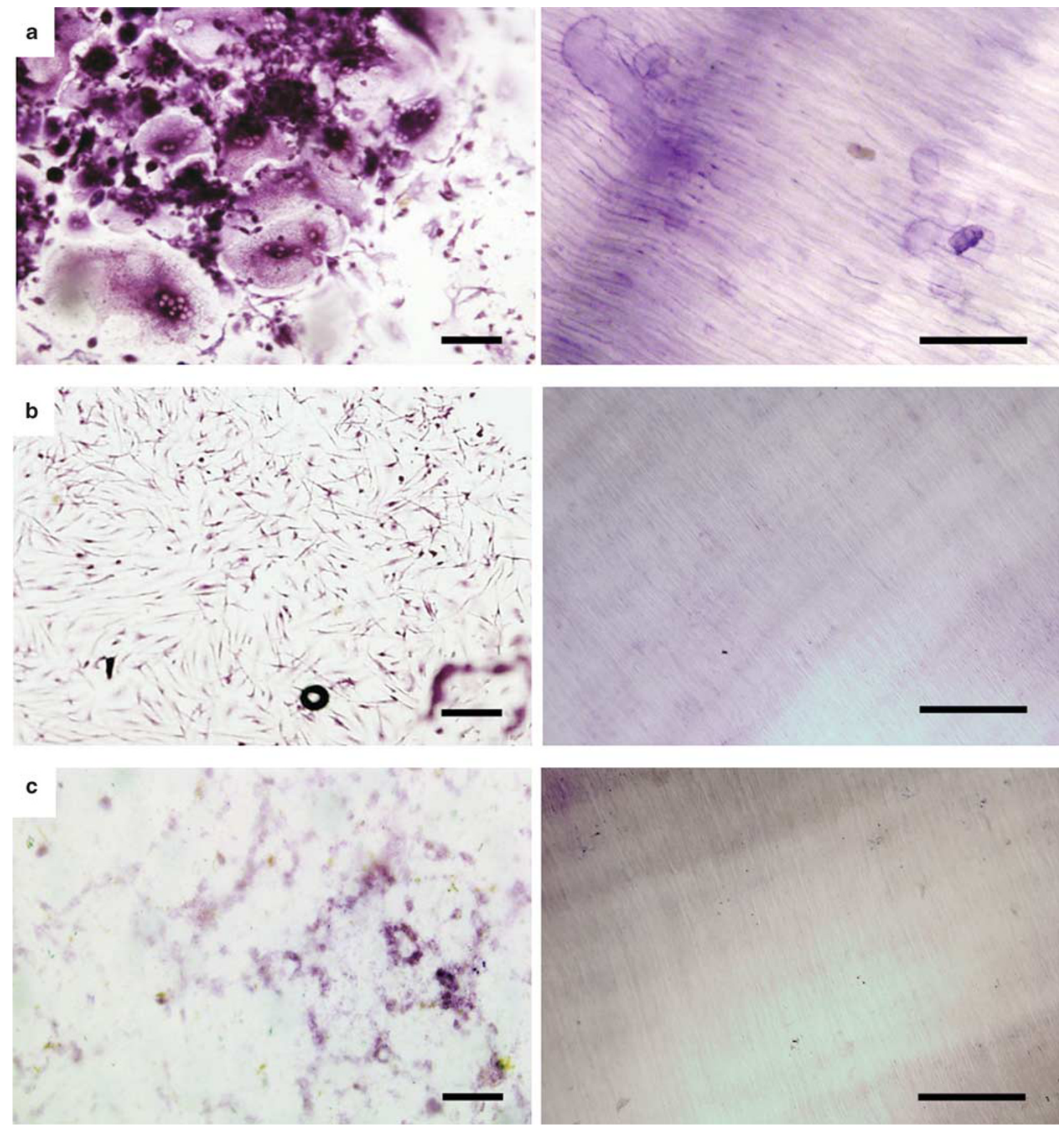

Figure 7 (a) M-CSF-primed CD14 ${ }^{+}$monocytes cultured with osteofibrous dysplasia-like adamantinoma stromal cells showing the formation of multinucleated TRAP $^{+}$cells. (b) No multinucleated TRAP ${ }^{+}$cells were observed in cultures containing M-CSF-primed $\mathrm{CD}_{14}{ }^{+}$monocytes alone (c) or M-CSF-primed osteofibrous dysplasia-like adamantinoma stromal cells alone. Scale bar is $100 \mu \mathrm{m}$.

and adamantinoma of long bones cells expressed mRNA for bone matrix proteins; however, neither adamantinoma of long bones nor osteofibrous dysplasia cells was independently capable of bonenodule formation. Both adamantinoma of long bones and osteofibrous dysplasia cells expressed RANKL, M-CSF and osteoclastogenic factors, which may contribute to the osteolysis associated with these lesions.
Expression of epithelial markers in cultured stromal cells from adamantinoma of long bones and osteofibrous dysplasia was similar with very few cells expressing EMA and cytokeratin. Epithelial conversion of mesenchymal cells is well recognised during embryological development and it has been proposed that this may have a role in adamantinoma of long bones tumourigenesis. ${ }^{16-18}$ It has been suggested that epithelial marker expression 
in some neoplasms of mesenchymal origin may result when tumour cells regress to a foetal state, where they are capable of expressing these markers. $^{19,20}$ This may explain why cytokeratin and EMA are found in a number of soft tissue tumours of mesenchymal origin. Other possible theories to account for the heterogenous expression of the epithelial elements in adamantinoma of long bones include implantation of epithelial cell nests in cortical bone, occurring either during embryological development or as a consequence of trauma, or epithelial differentiation of a multipotent mesenchymal cell. ${ }^{21-23}$ The majority of the cultured osteofibrous dysplasia and adamantinoma of long bones stromal cells in our study did not express cytokeratin and EMA. This would argue against implantation of epithelial elements and suggest that differentiation of tumour cells from a mesenchymal towards an epithelial phenotype is a possible cause of the EMA and cytokeratin expression in adamantinoma of long bones and osteofibrous dysplasia.

Bone formation is often a more prominent feature in osteofibrous dysplasia compared with adamantinoma of long bones. Histologically, osteofibrous dysplasia is composed of loose bundles of fibroblast-like cells among which are found trabeculae of woven bone lined by plump osteoblasts., ${ }^{3,22}$ There may be a zonal arrangement with progressive widening and increase in the number of mineralised bone trabeculae at the periphery of the lesion. As the trabeculae become more numerous, they fuse together and merge with the thickened cortical bone and there is centrifugal maturation of woven to lamellar bone. Cultured osteofibrous dysplasia stromal cells showed expression of alkaline phosphatase, a marker of the osteoblast phenotype. Adamantinoma stromal cells did not express alkaline phosphatase and both adamantinoma and osteofibrous dysplasia cells did not form bone nodules. Similar characteristics have been noted in the stromal cells of fibrous dysplasia, a lesion which has some morphological features in common with osteofibrous dysplasia. ${ }^{21,24,25}$ Osteofibrous dysplasia and adamantinoma of long bones stromal cells have been shown to differentially express markers of osteoblast differentiation, including osteocalcin, osteonectin and osteopontin; our RT-PCR findings confirm this observation that reflects the potential of stromal cells in these lesions to produce bone matrix proteins. ${ }^{26,27}$

Both adamantinoma and osteofibrous dysplasia cells were incapable of resorbing bone when cultured alone on dentine slices. These cells, however, expressed RANKL and, when cocultured with human monocytes, were capable of inducing the formation of multinucleated osteoclast-like $\mathrm{TRAP}^{+} / \mathrm{CD} 1^{+}$cells from these mononuclear phagocyte osteoclast precursors. This finding suggests that osteolysis in adamantinoma of long bones and osteofibrous dysplasia most likely occurs by RANKL-expressing mesenchymal stromal cells inducing macrophages in osteofibrous dysplasia and adamantinoma of long bones to undergo osteoclast differentiation, as occurs in other primary bone tumours. ${ }^{15,28,29}$

There are close similarities in tumour location and radiological features between osteofibrous dysplasia and adamantinoma of long bones. Our findings show that there are similarities and differences in stromal cell expression of epithelial and osteoblast markers in these lesions. On the basis of our findings, it is not possible to determine whether osteofibrous dysplasia represents a continuation of a reparative process associated with a pre-existing adamantinoma or whether osteofibrous dysplasia, differentiated adamantinoma and classic adamantinoma represent a continuum of lesions. However, our findings are in keeping with adamantinoma and osteofibrous dysplasia being closely related primary bone lesions. Taken together with the findings of previous studies, ${ }^{16,27}$ our results support the hypothesis of fibrous to epithelial transformation in osteofibrous dysplasia and adamantinoma of long bones, and suggest that the variable degree of bone formation seen in these two lesions reflects differences in osteoblast marker and bone matrix protein expression.

\section{Acknowledgements}

We would like to thank Chris Lowe for typing the manuscript and Arundhati Dongre for technical assistance. This study was supported by EuroBoNet, an EU-funded Network of Excellence, and the Rosetrees Charitable Trust. We would also like to acknowledge the support of the Oxford NIHR BRU.

\section{Discloser/conflict of interest}

The authors declare no conflict of interest.

\section{References}

1 Hogendoorn Pancras CW, , Hashimoto H. Adamantinoma. In: Fletcher C, Unni K, Mertens F (eds). Pathology and Genetics of Tumours of Soft Tissue and Bone. IARC: Lyon, 2002, pp 332-334.

2 Unni KK, Inwards YI, Bridge AN, et al. Tumors of the Bones and Joints AFIP Atlas of Tumor Pathology, 4th edn. AFIP: Washington DC, 2005, pp 281-298.

3 Campanacci M, Laus M. Osteofibrous dysplasia of the tibia and fibula. J Bone Joint Surg Am 1981;63: 367-375.

4 Benassi MS, Campanacci L, Gamberi G, et al. Cytokeratin expression and distribution in adamantinoma of the long bones and osteofibrous dysplasia of tibia and fibula. An immunohistochemical study correlated to histogenesis. Histopathology 1994;25:71-76.

5 Hazelbag HM, Fleuren GJ, Van den Broek LJCM, et al. Adamantinoma of the long bones: keratin subclass immunoreactivity pattern with reference to its histogenesis. Am J Surg Pathol 1993;17:1225-1233. 
6 Hazelbag HM, Wessels JW, Mollevangers P, et al. Cytogenetic analysis of adamantinoma of long bones: further indications for a common histogenesis with osteofibrous dysplasia. Cancer Genet Cytogenet 1997;97:5-11.

7 Ishida T, Iijima T, Kikuchi F, et al. A clinicopathological and immunohistochemical study of osteofibrous dysplasia, differentiated adamantinoma, and adamantinoma of long bones. Skeletal Radiol 1992;21:493-502.

8 Hazelbag HM, Taminiau AHM, Fleuren GJ, et al. Adamantinoma of the long bones. A clinicopathological study of thirty-two patients with emphasis on histological subtype, precursor lesion, and biological behavior. J Bone Joint Surg Am 1994;76:1482-1499.

9 Springfield DS, Rosenberg AE, Mankin HJ, et al. Relationship between osteofibrous dysplasia and adamantionma. Clin Orthop Relat Res 1994;308:234-244.

10 Czerniak B, Rojas-Corona RR, Dorfman HD. Morphologic diversity of long bone adamantinoma. The concept of differentiated (regressing) adamantinoma and its relationship to osteofibrous dysplasia. Cancer 1989;64:2319-2334.

11 Athanasou NA, Quinn J, McGee JO. Leucocyte common antigen is present on osteoclasts. J Pathol 1987;153:121-126.

12 Horton MA, Lewis D, McNulty K, et al. Monoclonal antibodies to osteoclastomas (giant cell bone tumors): definition of osteoclast-specific cellular antigens. Cancer Res 1985;45:5663-5669.

13 Beresford JN, Graves SE, Smoothy CA. Formation of mineralized nodules by bone derived cells in vitro: a model of bone formation? Am J Med Genet 1993;45: 163-178.

14 Gundle R, Beresford JN. The isolation and culture of cells from explants of human trabecular bone. Calcif Tissue Int 1995;56(Suppl 1):S8-S10.

15 Lau YS, Sabokbar A, Gibbons CL, et al. Phenotypic and molecular studies of giant-cell tumors of bone and soft tissue. Hum Pathol 2005;36:945-954.

16 Hazelbag HM, Van den Broek LJCM, Fleuren GJ, et al. Distribution of extracellular matrix components in adamantinoma of long bones suggests fibrous-to-epithelial transformation. Hum Pathol 1997;28:183-188.

17 Kuruc N, Franke WW. Transient coexpression of desmin and cytokeratins 8 and 18 in developing myocardial cells of some vertebrate species. Differentiation 1988;38:177-193.

18 Van Muijen GNP, Ruiter DJ, Warnaar SO. Coexpression of intermediate filament polypeptides in human-fetal and adulc tissues. Lab Invest 1987;57:359-369.

19 Ekblom P. Developmentally regulated conversion of mesenchyme to epithelium. FASEB J 1989;3: 2141-2150.

20 Tsarfaty I, Rong S, Resau JH, et al. The Met protooncogene mesenchymal to epithelial cell conversion. Science 1994;263:98-101.

21 Lichtenstein L. Dermal inclusion tumours in bone (socalled adamantinoma of limb bones). In: Lichtenstein L (ed). Bone Tumours. C.V. Mosby Co.: St Louis, 1997, pp 355-362.

22 Mirra JM. Adamantinoma and osteofibrous dysplasia. In: Mirra JM (ed). Bone Tumours. Clinical, Radiologic, and Pathologic Correlations (edn). Lea and Febiger: Philadelphia, 1989, pp 1204-1231.

23 Povysil C, Matejovsky Z. Ultrastructure of adamantinoma of long bones. Virchows Arch A Pathol Anat Histol 1981;393:233-244.

24 Riminucci M, Fisher LW, Shenker A, et al. Fibrous dysplasia of bone in the McCune-Albright syndrome: abnormalities in bone formation. Am J Pathol 1997;151:1587-1600.

25 Weiss SW, Dorfman HD. Adamantinoma of long bone. An analysis of nine new cases with emphasis on metastasizing lesions and fibrous dysplasia-like changes. Hum Pathol 1977;8:141-153.

26 Hopyan S, Gokgoz N, Bell RS, et al. Expression of osteocalcin and its transcriptional regulators corebinding factor alpha 1 and MSX2 in osteoid-forming tumours. J Orthop Res 1999;17:633-638.

27 Maki M, Athanasou N. Osteofibrous dysplasia and adamantinoma: correlation of proto-oncogene product and matrix protein expression. Hum Pathol 2004;35: 69-74.

28 Lau YS, Adamopoulos IE, Sabokbar A, et al. Cellular and humoral mechanisms of osteoclast formation in Ewing's sarcoma. Br J Cancer 2007;96:1716-1722.

29 Sun SG, Lau YS, Itonaga I, et al. Bone stromal cells in pagetic bone and Paget's sarcoma express RANKL and support human osteoclast formation. J Pathol 2006; 209:114-120. 